\title{
Improved COVID-19 ICU admission and mortality outcomes following treatment with statins: a systematic review and meta-analysis
}

\author{
Amir Vahedian-Azimi ${ }^{1}$, Seyede Momeneh Mohammadi², Farshad Heidari Beni ${ }^{3}$, Maciej Banach ${ }^{4,5,6}$, \\ Paul C. Guest ${ }^{7}$, Tannaz Jamialahmadi ${ }^{8,9}$, Amirhossein Sahebkar ${ }^{10,11,12}$
}

\author{
${ }^{1}$ Trauma Research Centre, Nursing Faculty, Baqiyatallah University of Medical \\ Sciences, Tehran, Iran \\ ${ }^{2}$ Department of Anatomical Sciences, Faculty of Medicine, Zanjan University \\ of Medical Sciences, Zanjan, Iran \\ ${ }^{3}$ Nursing Care Research Center (NCRC), School of Nursing and Midwifery, \\ Iran University of Medical Sciences, Tehran, Iran \\ ${ }^{4}$ Department of Hypertension, Chair of Nephrology and Hypertension, \\ Medical University of Lodz, Lodz, Poland \\ ${ }^{5}$ Polish Mother's Memorial Hospital Research Institute (PMMHRI), Lodz, Poland \\ ${ }^{6}$ Cardiovascular Research Centre, University of Zielona Gora, Zielona Gora, Poland \\ ${ }^{7}$ Laboratory of Neuroproteomics, Department of Biochemistry and Tissue Biology, \\ Institute of Biology, University of Campinas (UNICAMP), Campinas, Brazil \\ ${ }^{8}$ Department of Food Science and Technology, Quchan Branch, Islamic Azad \\ University, Quchan, Iran \\ ${ }^{9}$ Department of Nutrition, Faculty of Medicine, Mashhad University of Medical Sciences, \\ Mashhad, Iran \\ ${ }^{10}$ Biotechnology Research Centre, Pharmaceutical Technology Institute, \\ Mashhad University of Medical Sciences, Mashhad, Iran \\ ${ }^{11}$ Biomedical Research Centre, Mashhad University of Medical Sciences, Mashhad, \\ Iran \\ ${ }^{12}$ School of Pharmacy, Mashhad University of Medical Sciences, Mashhad, Iran
}

Submitted: 29 January 2021; Accepted: 9 February 2021;

Online publication: 10 February 2021

Arch Med Sci 2021; 17 (3): 579-595

DOI: https://doi.org/10.5114/aoms/132950

Copyright (c) 2021 Termedia \& Banach

\section{Abstract}

Introduction: Approximately $1 \%$ of the world population has now been infected by the severe acute respiratory syndrome coronavirus 2 (SARS-CoV-2), which causes coronavirus disease 2019 (COVID-19). With cases still rising and vaccines just beginning to rollout, we are still several months away from seeing reductions in daily case numbers, hospitalisations, and mortality. Therefore, there is a still an urgent need to control the disease spread by repurposing existing therapeutics. Owing to antiviral, anti-inflammatory, immunomodulatory, and cardioprotective actions, statin therapy has been considered as a plausible approach to improve COVID-19 outcomes.

Material and methods: We carried out a meta-analysis to investigate the effect of statins on 3 COVID-19 outcomes: intensive care unit (ICU) admission, tracheal intubation, and death. We systematically searched the PubMed, Web of Science, Scopus, and ProQuest databases using keywords related to our aims up to November 2, 2020. All published observational studies and randomised clinical trials on COVID-19 and statins were retrieved. Statistical analysis with random effects modelling was performed using STATA16 software.

Results: The final selected studies ( $n=24$ studies; 32,715 patients) showed significant reductions in ICU admission $(\mathrm{OR}=0.78,95 \% \mathrm{Cl}: 0.58-1.06 ; n=10$; $\left.1^{2}=58.5 \%\right)$ and death (OR $\left.=0.70,95 \% \mathrm{Cl}: 0.55-0.88 ; n=21 ; P^{2}=82.5 \%\right)$ outcomes, with no significant effect on tracheal intubation $(\mathrm{OR}=0.79 ; 95 \% \mathrm{Cl}$ : $\left.0.57-1.11 ; n=7 ; I^{2}=89.0 \%\right)$. Furthermore, subgroup analysis suggested

\author{
Corresponding author: \\ Prof. Amirhossein Sahebkar \\ Biotechnology Research Center \\ Pharmaceutical Technology \\ Institute \\ Mashhad University of \\ Medical Sciences \\ Mashhad 9177948564, Iran \\ Phone: +985138002299 \\ Fax: +985138002287 \\ E-mails: \\ sahebkara@mums.ac.ir; \\ amir_saheb2000@yahoo.com
}


that death was reduced further by in-hospital application of stains (OR $=0.40,95 \% \mathrm{Cl}: 0.22-0.73, n=3$; $\left.r^{2}=82.5 \%\right)$, compared with pre-hospital use (OR $\left.=0.77,95 \% \mathrm{Cl}: 0.60-0.98, n=18 ; I^{2}=81.8 \%\right)$.

Conclusions: These findings call attention to the need for systematic clinical studies to assess both pre- and in-hospital use of statins as a potential means of reducing COVID-19 disease severity, particularly in terms of reduction of ICU admission and total mortality reduction.

Key words: coronavirus, COVID-19, disease severity, intensive care unit, mortality, statins.

\section{Introduction}

As of December 2020, almost 80 million people have been infected by the severe acute respiratory syndrome coronavirus 2 (SARS-CoV-2), which causes coronavirus disease 2019 COVID-19 [1, 2]. This equates to more than $1 \%$ of the world population [3]. The total number of COVID-19-related deaths has now reached more than 1.7 million, which translates to a rate of $2.2 \%$ of the cases. In the face of this, the last several weeks have seen the rollout of new vaccines for protection against COVID-19, which have been produced in record time due to an unprecedented worldwide effort [4-6]. Although massive worldwide networks have already been put in place to distribute these vaccines across the globe, we are still several months away from seeing a drop in daily COVID-19 case numbers, hospitalisations, and mortality. Therefore, there is still an urgent need to control the disease spread by testing of new drugs and compounds and by repurposing existing therapeutics $[7,8]$.

Epidemiological studies have shown that age and preexisting medical conditions such as hypertension, cardiopulmonary diseases, diabetes and obesity are associated with a more severe disease course and higher mortality in patients hospitalized with COVID-19 disease [9-13]. However, it is still not known why some of these individuals experience a more severe form of the diseasewhile others in the same high-risk groups only show mild symptoms or are asymptomatic. One possible explanation is due to the protective effects of medications routinely used to treat these conditions, although this has been debated $[14,15]$.

Statins are inexpensive, easily available, and are already in widespread use for treatment of cardiovascular disorders, which has created considerable interest in further exploration of their repurposing as a new treatment for managing the severity of COVID-19 infections [16-21]. Statins are known to reduce the risk of major cardiovascular events and to counteract inflammation, the immune response, and oxidative stress damage [22-25], which may reduce the risk of a more severe disease course and improve outcomes in COVID-19 patients. However, it should be emphasised that there is still a lack of clinical consensus on the efficacy of statins in improving disease outcomes in COVID-19 patients, particularly with respect to whether or not the drug has been administered before or after diagnosis.

To address these points, we have carried out a systematic review and meta-analysis to investigate the effect of statins on COVID-19 disease outcomes. In particular, we focused on the rate of intensive care unit (ICU) admission, the need for mechanical ventilation, and mortality. It was also of special interest to determine whether or not there was a difference in these outcomes in patients who had received statins before or after their COVID-19 diagnosis.

\section{Material and methods}

\section{Search strategy}

This systematic review followed the Preferred Reporting Items for Systematic Reviews and Meta-Analyses (PRISMA) guidelines. Ethical approval was obtained from the research ethics committee of Baqiyatallah University of Medical Sciences with the ethics code of IR.BMSU. REC.1399.009 in 2020-03-26. The searches were conducted in the Web of Science, PubMed, Scopus, and ProQuest databases for eligible articles up to November 2, 2020, with no restrictions on language or publication date. The reference lists of articles were also reviewed using forward and backward citation tracking to identify other eligible documents. The population, intervention, comparison, and outcome (PICO) criteria were formulated as follows:

- population: patients infected with SARS-CoV-2 confirmed by reverse transcriptase polymerase chain reaction (RT-PCR);

- intervention: statin therapy;

- comparison: patients infected with SARS-CoV-2 who did not use statins;

- outcomes: intensive care unit (ICU) admission, tracheal intubation, and all-cause mortality.

The overall aim was to determine whether or not a relationship exists between statin therapy and improvement of outcomes in patients infected with SARS-CoV-2. The following is an example of the PubMed title and abstract search strategy:

- novel coronavirus OR novel coronavirus 2019 OR 2019 novel coronavirus OR 2019 nCoV OR Wuhan coronavirus OR Wuhan pneumonia OR covid-19 OR 2019-nCoV OR SARS-CoV-2 OR coronavirus 2019 OR 2019-nCoV 
AND

- statin OR HMGCoA reductase inhibitor OR lovastatin OR fluvastatin OR pravastatin OR rosuvastatin $O R$ pitavastatin $O R$ atorvastatin $O R$ simvastatin OR cerivastatin OR lipitor OR lescol OR lecol AND xl OR mevacor OR altoprev OR pravachol OR crestor OR zocor OR livalo.

To ensure comprehensive searches of all articles related to the effect of statins on outcomes, we followed a search strategy without considering specific outcomes. After finding all relevant articles, we concluded that only 3 outcomes were amenable to meta-analysis due to sufficient numbers of studies including the parameters in the literature: 1) ICU admission; 2) tracheal intubation; and 3) allcause mortality. Therefore, the study continued with a focus on these 3 outcomes.

\section{Inclusion and exclusion criteria}

The inclusion criteria were as follows:

- observational studies (case-control studies and cohort studies) and randomised clinical trials evaluating the impact of statins on patients with COVID-19 in either prospective or retrospective formats;

- outcome measures that included all-cause mortality, ICU admission, and tracheal intubation. The exclusion criteria were as follows:

- clinical case reports, literature reviews, animal studies, and studies involving in vitro experiments;

- studies not including statin non-users.

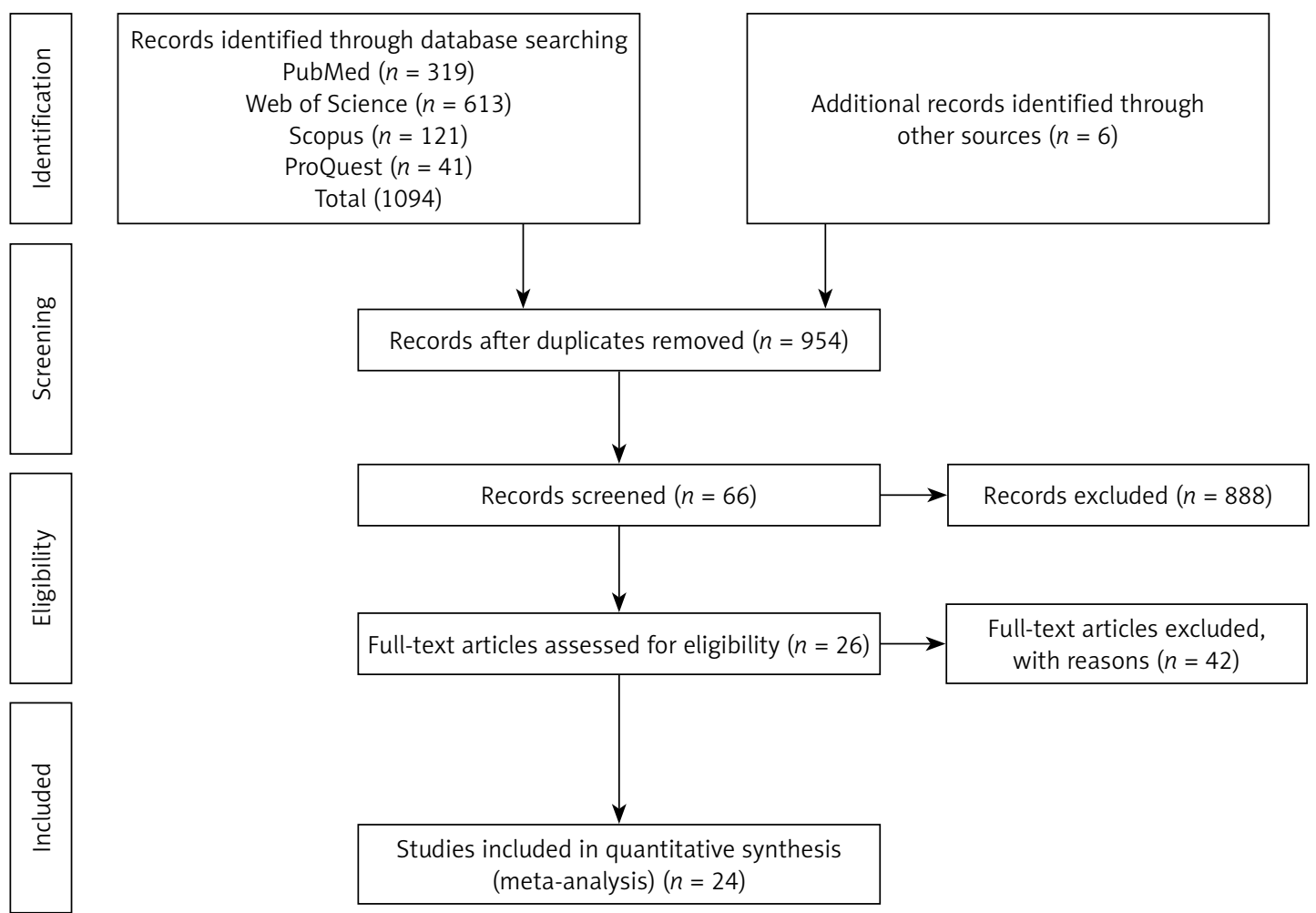

\section{Study selection}

We performed a 4-step process carried out in parallel by 2 authors (AHS and AVA) to determine eligible criteria for inclusion. First, we searched the PubMed, Web of Science, Scopus, and ProQuest studies. Six additional studies were identified via Internet searches, bringing the total number of articles to 1100 . Next, we exported the identified records into the EndNote X9 software, reviewed the full list, and excluded duplicate studies, leaving 954 records. The titles and abstracts of these articles were then screened, and relevant studies selected for full text evaluation. The full texts of the 66 remaining studies were assessed for eligibility, leaving 26 studies. Finally, 24 studies that met all inclusion and exclusion criteria were selected for the analysis (Figure 1).

\section{Data extraction}

Data extraction was completed independently by 2 researchers (FHB and AVA), and the data form was populated to include the following: authors name, sample size, subjects, reported rate, setting, study design, result, and conclusions (the year was not included because all studies were from 2020). The main outcomes were also summarised. Discrepancies in extracted data were resolved by discussion between authors. Remaining disagreements were resolved by a third reviewer. The extracted data are displayed in Table I. databases and identified 1094 potentially relevant

Figure 1 . Study selection process 
Amir Vahedian-Azimi, Seyede Momeneh Mohammadi, Farshad Heidari Beni, Maciej Banach, Paul C. Guest, Tannaz Jamialahmadi, Amirhossein Sahebkar

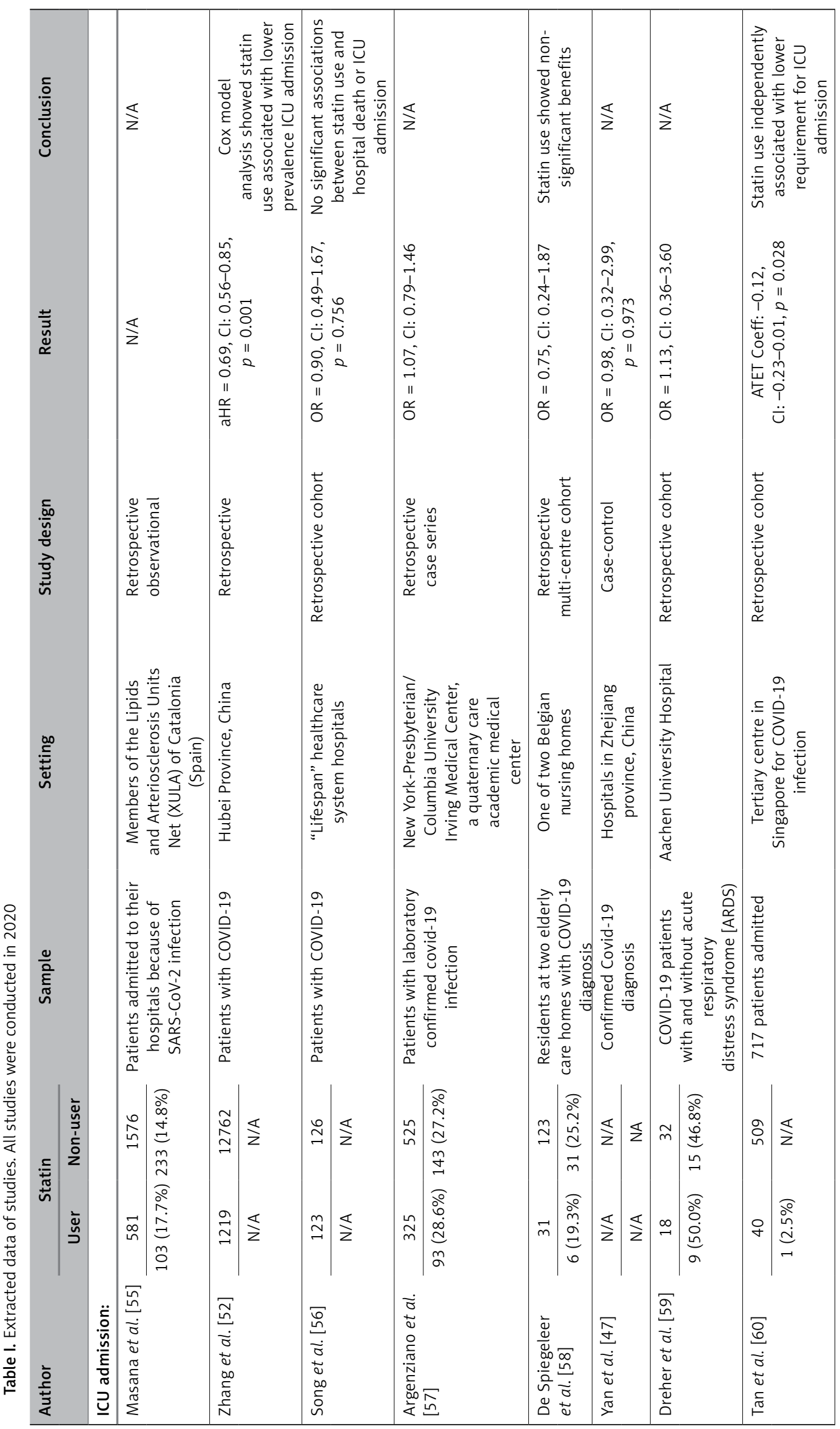




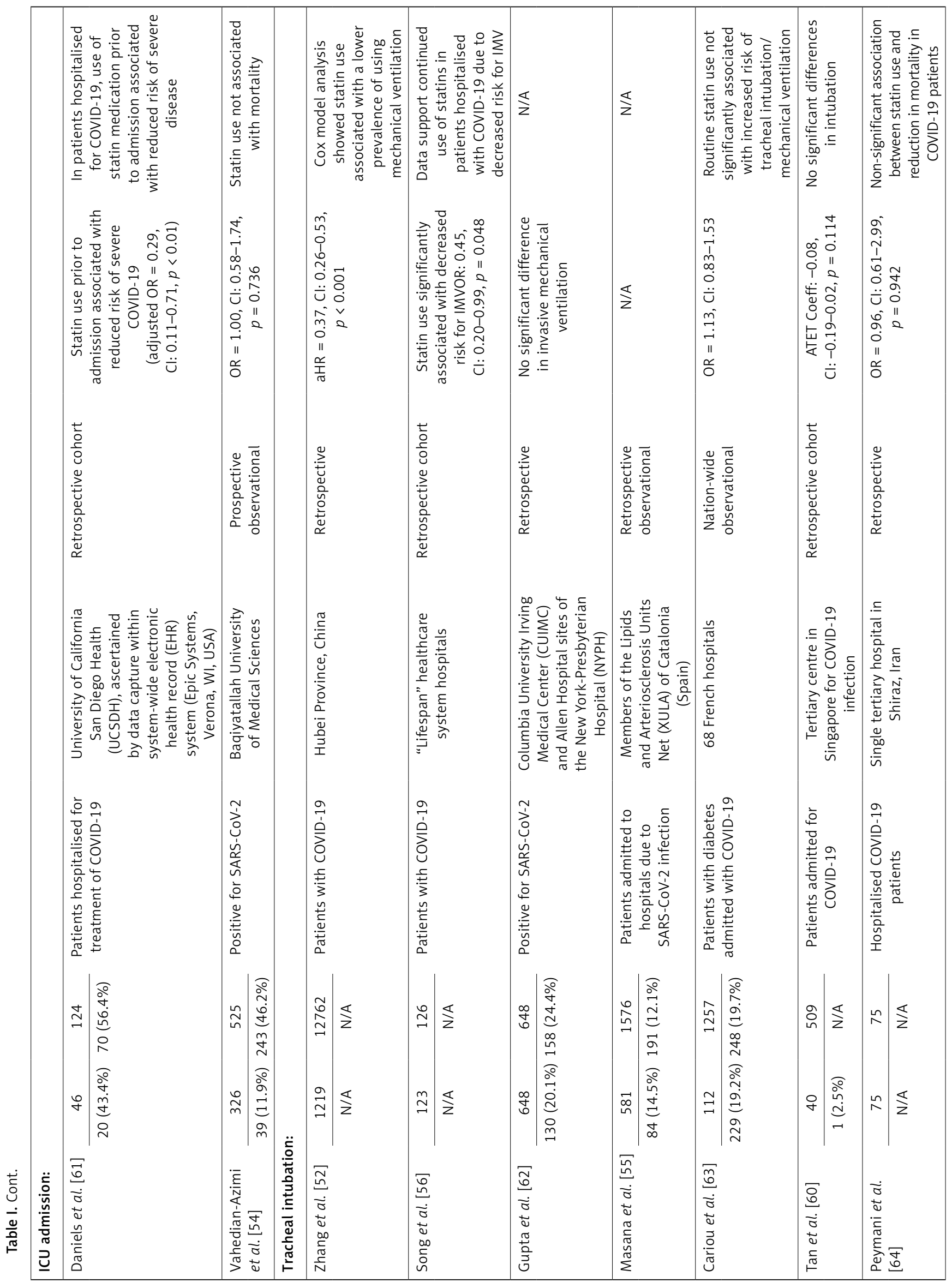


Amir Vahedian-Azimi, Seyede Momeneh Mohammadi, Farshad Heidari Beni, Maciej Banach, Paul C. Guest, Tannaz Jamialahmadi, Amirhossein Sahebka

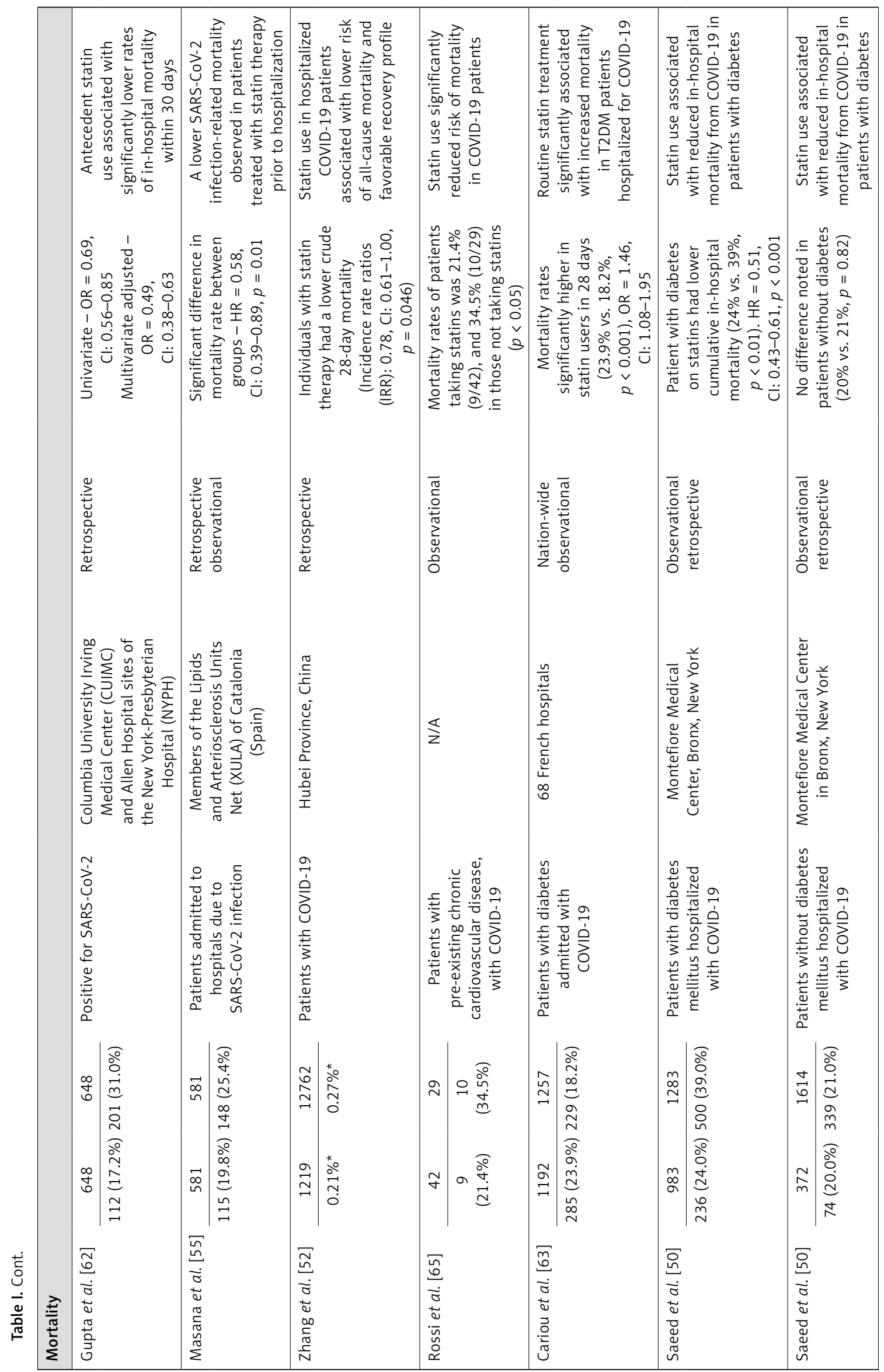




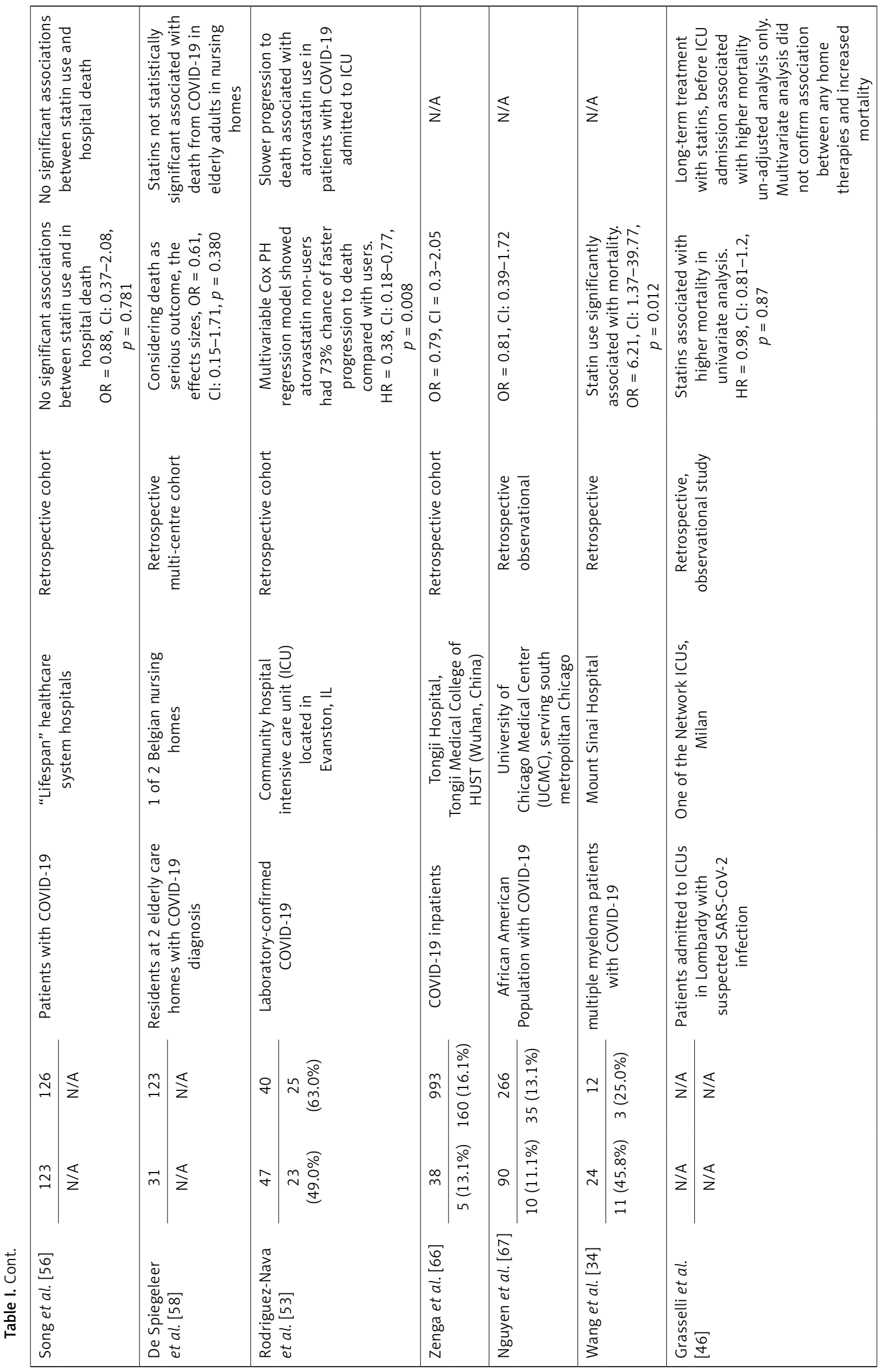


Amir Vahedian-Azimi, Seyede Momeneh Mohammadi, Farshad Heidari Beni, Maciej Banach, Paul C. Guest, Tannaz Jamialahmadi, Amirhossein Sahebkar

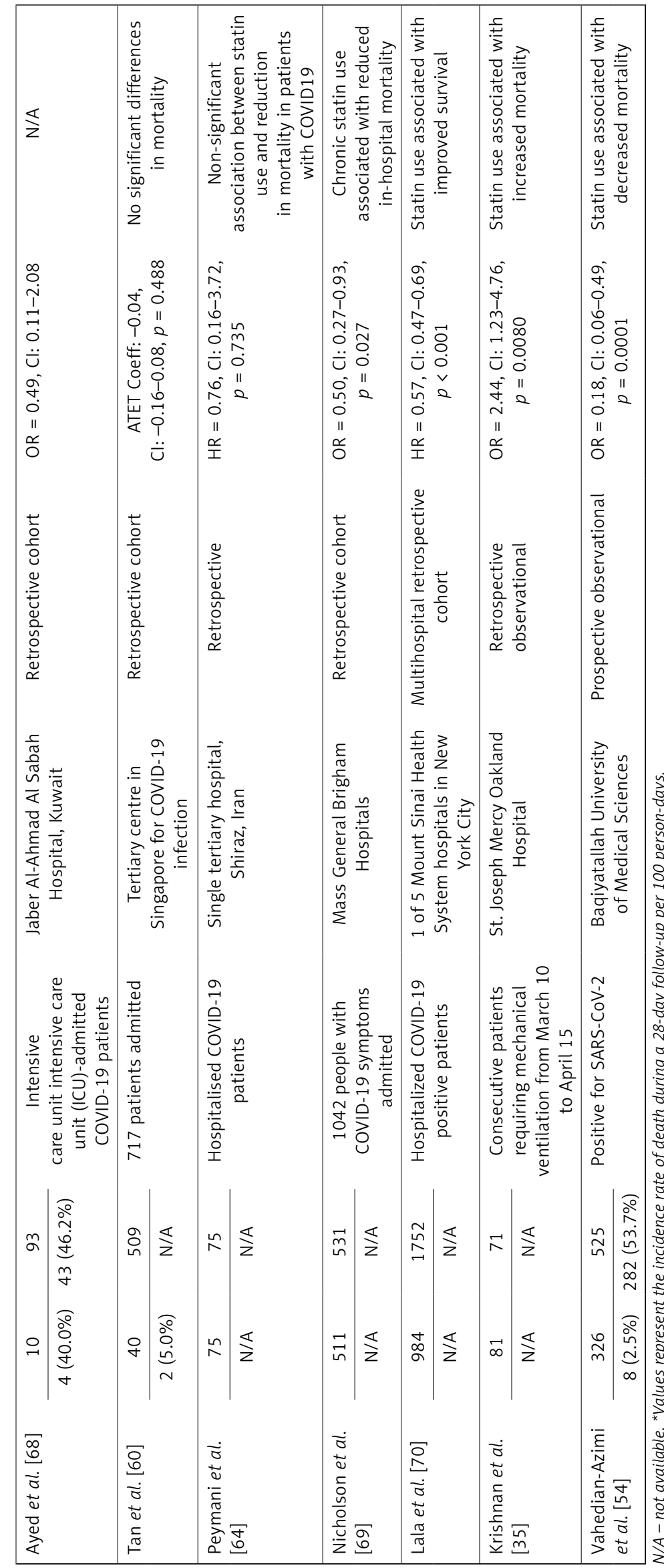




\section{Quality assessment}

The methodological quality assessment of studies was performed independently by 2 authors (FHB and AVA) using the Newcastle-Ottawa Scale (NOS) for cohort studies, and disagreements were resolved by discussion between the authors. The NOS scale was developed to assess the quality of nonrandomised studies with respect to design, content, and ease of use in the interpretation of results of meta-analyses. Using this scale, each study was assessed according to 3 broad perspectives: 1) selection of the study groups; 2) comparability of the groups; and 3) the ascertainment of either the exposure or outcome of interest for case-control or cohort studies, respectively [26]. A rating system of 0-3 stars per category was used to indicate quality, giving a range of 0-9 total stars per study [27]. Disagreements about inclusion criteria, data extraction, and quality assessment were resolved by consensus. The quality assessment of the studies is shown in Table II.

\section{Statistical analysis}

All analyses were conducted using STATA16 (StataCorp, College Station, Texas, USA). The reporting of the study was adapted based on the PRISMA statement [28]. Data extraction for the main outcomes was conducted and random effects metaanalyses were carried out using the restricted maximum likelihood method [29]. The random effects model was applied due to the possible existence of other unknown, unregistered, or unpublished studies, which we could not access. Between-study heterogeneity was evaluated using Cochran Q test, tau-squared $\left(t^{2}\right)$, H-squared $\left(H^{2}\right)$, and $l$-squared $\left(I^{2}\right)$ statistics. The significance of the results of the tests and values higher than $75 \%$ for $R$ were considered as having substantial heterogeneity, while $H^{2}=1$ was taken to represent perfect homogeneity among the studies [30]. To assess the publication bias, funnel plots, regression-based Egger's [31], and nonparametric rank correlation-based Begg's [32] tests were used to test for the presence of small-study effects often associated with publication bias. A funnel plot is a scatter plot of study-specific effect sizes on the $x$ axis against standard errors on the $y$ axis. In the absence of small-study effects, the plot should look symmetrical. A nonparametric trim-and-fill method of accounting for publication bias was conducted, and the modified effect size was estimated after adjusting for publication bias. This estimates the number of studies potentially missing from a meta-analysis because of publication bias, imputes these studies, and computes the overall effect-size estimate using the observed and imputed studies. This can also be used to generate a funnel plot, in which omitted studies are imputed [33]. Common effect sizes were calculated as an odds ratio (OR) with 95\% confidence interval (CI) for each main outcome, and the results were presented using forest plots. The individual and the overall effect sizes, Cls, heterogeneity statistics, and significance tests for effect size were reported. Additionally, predetermined subgroup analyses were conducted according to the conditions of either in- or pre-hospital use of the statin treatments.

\section{Results}

\section{ICU admission}

The OR from 10 studies was $0.78(95 \% \mathrm{Cl}$ : $0.58-1.06, p=0.11$ ), with significant heterogeneity between studies $\left(\tau^{2}=0.16, l^{2}=85.80 \%\right.$, $\left.H^{2}=7.04, Q_{(d f=9)}=107.72, p_{Q}<0.001\right)$ (Figure $2 \mathrm{~A}$ ). Assessment for bias by Egger's $(p=0.815)$ and Begg's $(p=0.142)$ tests showed no significant small-study effects. Further visual inspection of the funnel plot suggested a slight degree of publication bias (Figure $2 \mathrm{~B}$ ). Extending the results of the analyses for the nonparametric trim-and-fill method of accounting for publication bias showed that there should be 2 additional studies for inclusion (Figure $2 \mathrm{C}$ ). A re-estimation of the overall OR after these studies was included, which resulted in an $\mathrm{OR}=0.70(95 \% \mathrm{Cl}: 0.52-0.94)$.

\section{Tracheal intubation}

The OR from 7 studies was $0.79(95 \% \mathrm{Cl}$ : $0.57-1.11, p=0.18$ ) based on the random effects model analysis, with significant heterogeneity between studies $\left(\tau^{2}=0.16, I^{2}=88.99 \%, H^{2}=9.08\right.$, $Q_{(d f=6)}=34.9, p_{Q}<0.001$ ) (Figure $3 \mathrm{~A}$ ). Assessment for bias by Egger's $(p=0.447)$ and Begg's $(p=0.115)$ tests showed no significant small-study effects, and visual inspection of the funnel plot suggested no publication bias (Figure $3 \mathrm{~B}$ ). Therefore, we did not extend the results of the analyses for nonparametric trim and fill method of accounting for publication bias in this case.

\section{Death}

The OR from 21 studies which assessed the effect of statins on all-cause mortality was 0.70 (95\% Cl: $0.55-0.88, p<0.01)$ based on a random effects model, with significant heterogeneity between studies $\left(\tau^{2}=0.18, l^{2}=82.55 \%, H^{2}=5.73\right.$, $\left.Q_{(d f=20)}=97.04, p_{Q}<0.001\right)$. Further investigation detected 2 outliers with a large effect size [34, 35]. We removed these from further analyses for this outcome, which resulted in a slight reduction in effect size $(\mathrm{OR}=0.64,95 \% \mathrm{Cl}$ : 0.52-0.79, $p<0.01)$; although heterogeneity was reduced, it still remained significant $\left(\tau^{2}=0.11\right.$, $\left.I^{2}=76.50 \%, H^{2}=4.23, Q_{(d f=18)}=76.5, p_{Q}<0.001\right)$ 
Amir Vahedian-Azimi, Seyede Momeneh Mohammadi, Farshad Heidari Beni, Maciej Banach, Paul C. Guest, Tannaz Jamialahmadi, Amirhossein Sahebkar

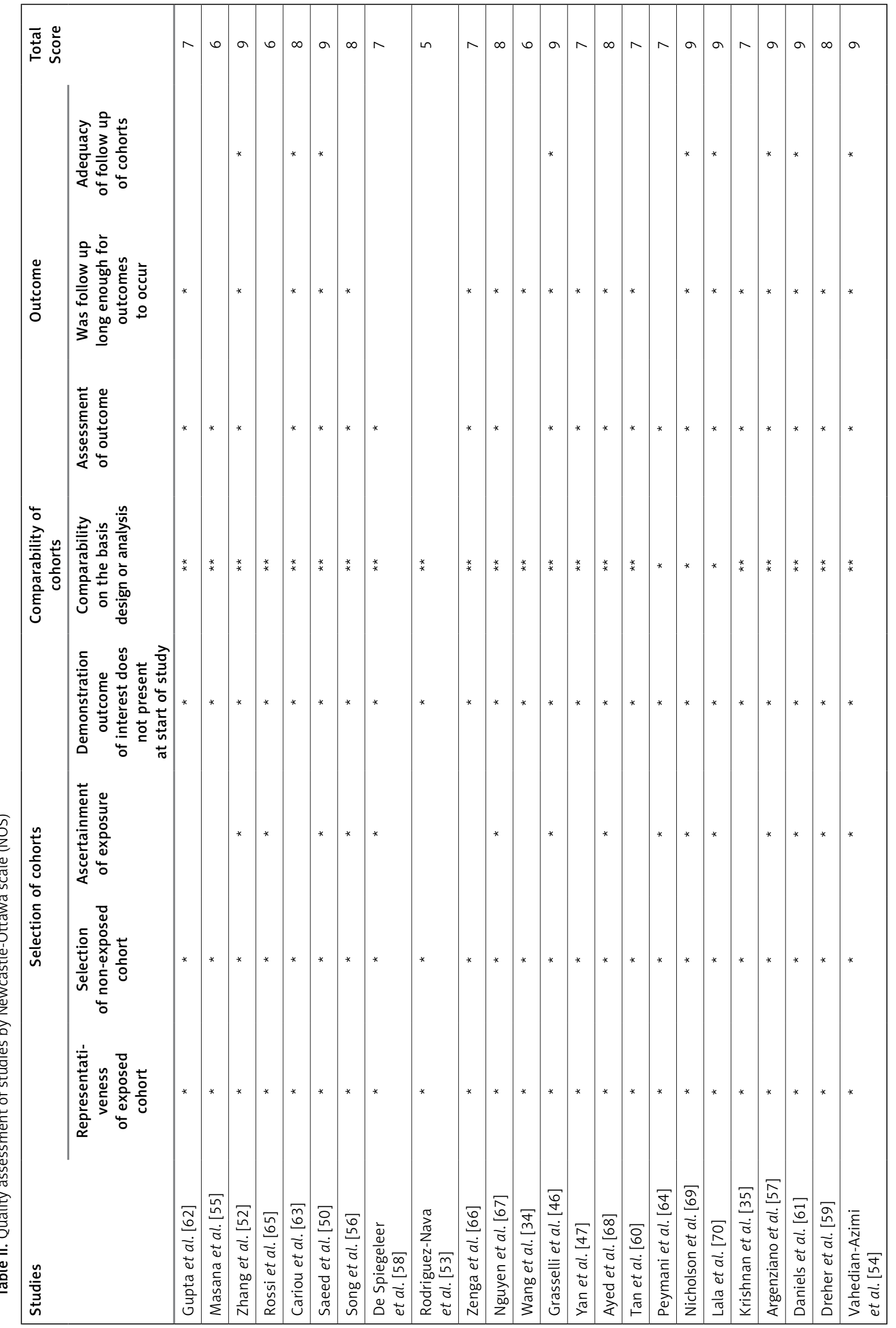


A

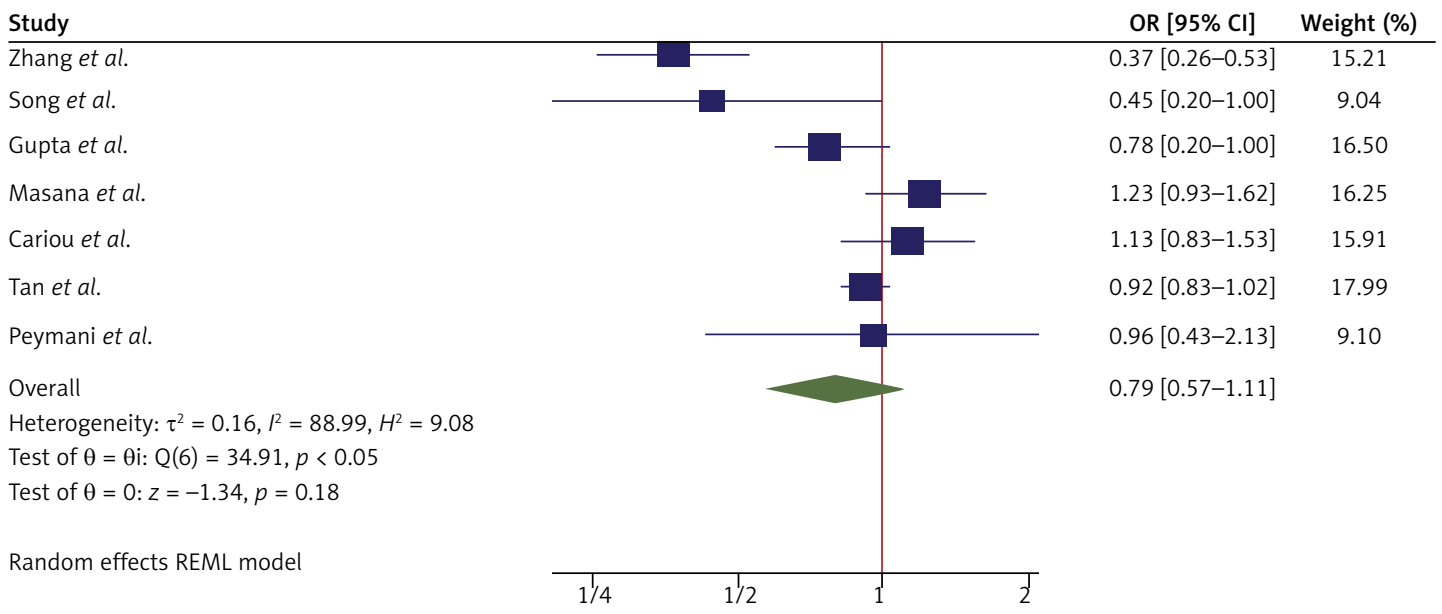

B

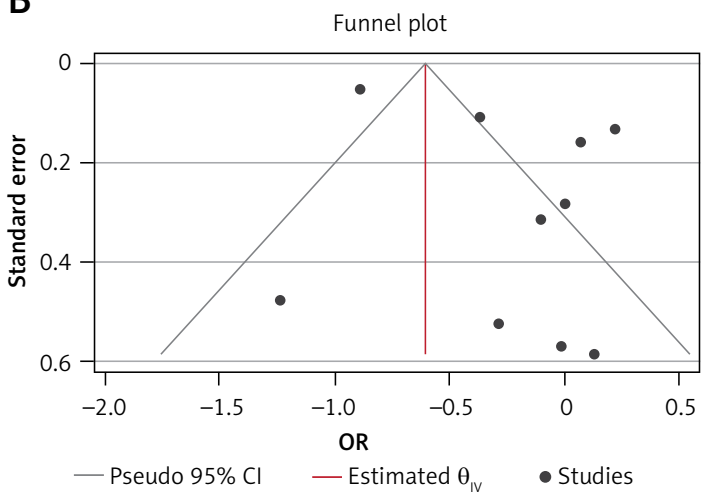

C

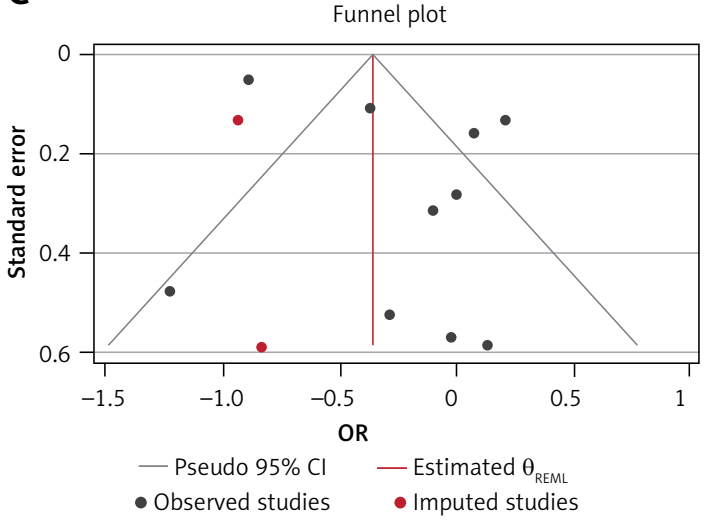

Figure 2. A - Forest plot of individual effect sizes within each study for ICU admission outcome. The overall effect is shown by the green symbol. B - Funnel plot showing publication bias for ICU admission outcome. C - Extending the results using the nonparametric trim and fill method to account for publication bias. Two additional studies were included in this analysis (shown in red)

(Figure 4 A). Assessment for bias by Egger's $(p=0.903)$ and Begg's $(p=0.349)$ tests showed no significant small-study effects, and visual inspection of the funnel plot suggested no publication bias (Figure $4 \mathrm{~B}$ ).

\section{Subgroup analysis}

The forest plot of individual ORs of the predetermined subgroup analyses indicated a significant effect in the pre-hospital use (OR $=0.70$, $95 \% \mathrm{Cl}: 0.56-0.86, n=18)$ and the in-hospital use $(\mathrm{OR}=0.40,95 \% \mathrm{Cl}: 0.22-0.73, n=3)$ subgroups, with a non-significant difference between the subgroups $\left(Q_{(d f=1)}=2.86, p_{Q}=0.09\right)$ (Figure $5 \mathrm{C}$ ). Additionally, although the heterogeneity was reduced slightly in the 2 subgroups, the amount of reduction was not considerable and remained significant in the pre-hospital group $(p<0.05)$. The heterogeneity was not significant in the in-hospital group ( $p=0.08)$, which may be due to the smaller number of studies in this subgroup and thus a lower power of the heterogeneity test.

\section{Discussion}

Overall, this systematic review and meta-analysis shows a significant benefit of statin use in reducing the severity of COVID-19 disease. We found a $22-30 \%$ reduction of the risk of ICU admission and a $30 \%$ reduction in the risk of death due to COVID-19 disease. In addition, our subgroup analysis showed that in-hospital use of statins was associated with as much as a $60 \%$ reduction in mortality while pre-hospital use was associated with a $30 \%$ reduction, although the difference between the subgroups did not reach significance. Statin use was not associated with the tracheal intubation outcome.

Statins may help by preventing the development of severe lung injury and acute respiratory distress syndrome (ARDS) by protecting against inflammation via modulation of cytokine over-expression, angiotensin-converting enzyme 2 (ACE2) expression, and the immune response in COVID-19 patients $[18,36,37]$. Statins have been shown to inhibit nuclear factor $\kappa \mathrm{B}(\mathrm{NF}-\kappa \mathrm{B})$, which mediates inflam- 
A

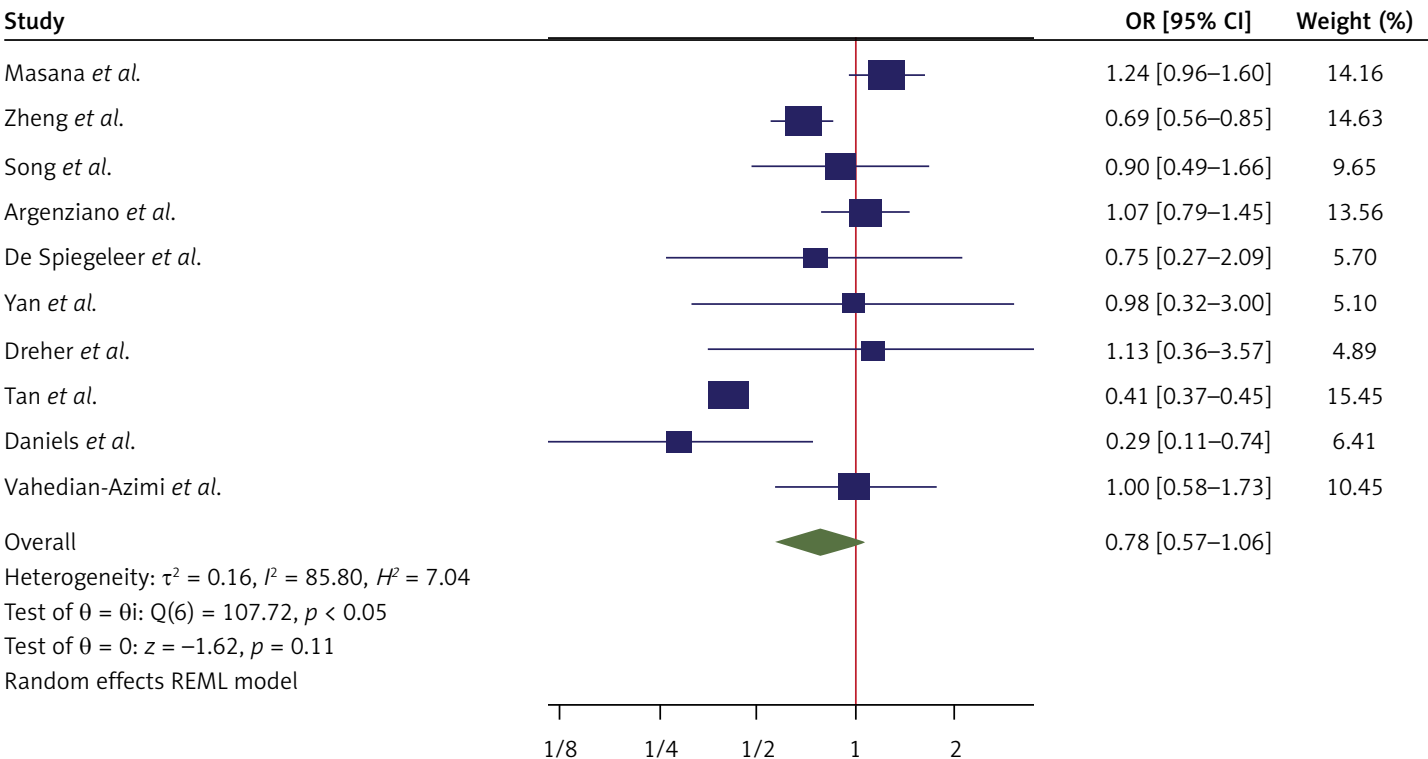

B

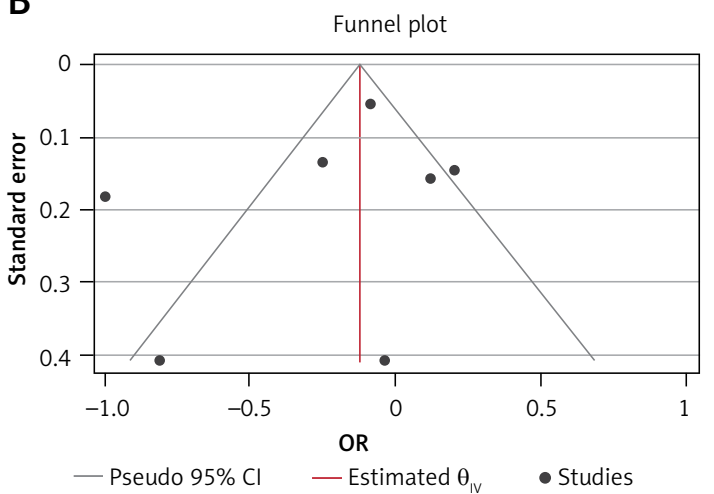

matory responses during infections [38, 39]. Statins may also be used to counteract the cytokine storm, which can also occur in severe cases of viral infection such as in COVID-19 cases [40, 41]. These properties support statin continuation for mitigating ARDS and multiorgan failure, which can occur in the acute phases of COVID-19 illness [42]. In addition, statins can exert direct antiviral effects [43]. We recently produced in-silico evidence showing that statins can bind with high affinity to the main Mpro protease, which mediates viral replication and transcription and could potentially exert anti-SARS-CoV-2 activity through inhibition of viral replication [44]. Statins also exert anti-thrombotic actions through modulation of endothelial cell activation and platelet aggregation, and thereby mitigate coagulopathies associated with infection, the cytokine storm, and impending organ failure $[18,19]$ (Figure 5).

Our findings regarding the reduced deaths in COVID-19 patients using statins are consistent with mechanistic findings on the anti-thrombotic, anti-inflammatory, antiviral, and immunomodulatory actions of statins $[18,43]$ as well as those of a recent meta-analysis of 6 studies, which found
Figure 3. A - Forest plot of individual effect sizes within each study for tracheal intubation outcome. The overall effect is shown by the green symbol. B - Funnel plot showing publication bias for tracheal intubation outcome

a pooled $30 \%$ risk reduction of severe disease or mortality in COVID-19 patients taking statins [45]. However, 2 of the studies within the previous analyses based statin use on historical record with no mention of pre- or in-hospital administration $[46,47]$. Differences in the type of statin used may also account for discrepancies across different studies. This possibility was highlighted in a study by Rossi et al., which showed that administration of simvastatin and atorvastatin reduced mortality in COVID-19 patients, whereas those treated with pravastatin and rosuvastatin showed no such difference [48]. In contrast, the CORONADO study found that statin use was associated with an increased risk of death in diabetes patients with COVID-19 [49], although this conflicted with another study, which found that statin application reduced mortality in COVID-19 diabetic patients [50] These discrepancies may be due to the use of different statins, because the statin type was not listed in the former study and the latter mainly involved the use simvastatin and atorvastatin.

Another possibility for discrepancies in the prehospital use of statins with respect to COVID-19 
A

Study

OR $[95 \% \mathrm{Cl}] \quad$ Weight $(\%)$

Pre-hospital

Gupta et al.

Masana et al.

Rossi et al.

Cariou et al.

Saeed et al.

Saeed et al. (b)

Song et al.

De Spiegeleer et al.

Zeng et al.

Nguyen et al.

Wang et al.

Grasselli et al.

Ayed et al.

Tan et al.

Peymani et al.

Nicholson et al.

Lala et al.

Krishnan et al.

Heterogeneity: $\tau^{2}=0.16, R^{2}=81.77 \%, H^{2}=5.48$

Test of $\theta=\theta \mathrm{i}: \mathrm{Q}(17)=86.87, p<0.05$

In-hospital

Zhang et al.

Rodriguez-Nava et al.

Vahedian-Azimi et al.

Heterogeneity: $\tau^{2}=0.17, P^{2}=61.00 \%, H^{2}=2.56$

Test of $\theta=\theta \mathrm{i}: \mathrm{Q}(2)=5.06, p=0.08$

Overall

Heterogeneity: $\tau^{2}=0.18, I^{2}=82.55 \%, H^{2}=5.73$

Test of $\theta=\theta \mathrm{i}: \mathrm{Q}(20)=97.04, p<0.05$

Test of group differences: $\mathrm{Qb}(1)=3.87, p=0.05$

Random effects model

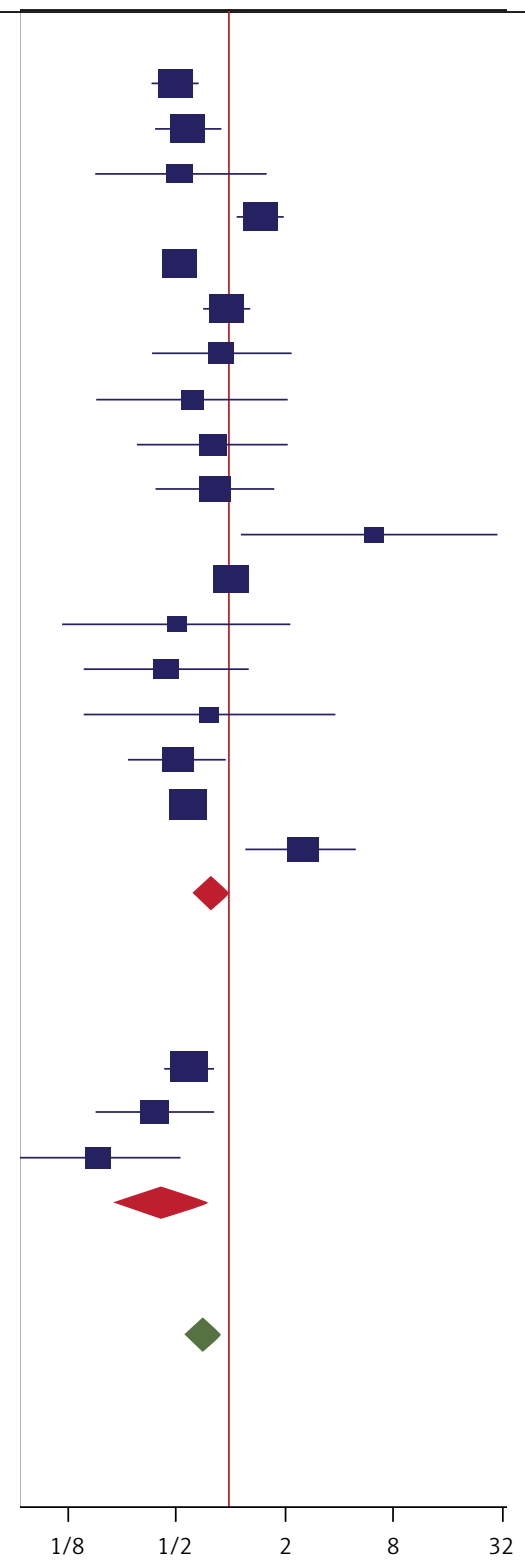

$0.49[0.38-0.63] \quad 7.24$

$0.58[0.38-0.88] \quad 6.35$

$0.52[0.18-1.53] \quad 2.95$

$1.46[1.09-1.96] \quad 7.02$

$0.51[0.43-0.61] \quad 7.58$

$0.93[0.70-1.24] \quad 7.07$

0.88 [0.37-2.09] 3.81

$0.61[0.18-2.06] \quad 2.52$

0.79 [0.30-2.07] $\quad 3.39$

0.81 [0.39-1.70] 4.41

$6.21[1.15-33.5] \quad 1.55$

0.98 [0.81-1.19] 7.49

0.49 [0.11-2.13] $\quad 1.92$

$0.43[0.15-1.21] \quad 3.11$

$0.76[0.16-3.66] \quad 1.73$

$0.50[0.27-0.93] \quad 5.10$

$0.57[0.47-0.69] \quad 7.51$

$2.44[1.24-4.80] \quad 4.76$

$0.77[0.60-0.98]$

$0.58[0.43-0.79] \quad 6.94$

0.38 [0.18-0.79] 4.49

0.18 [0.06-0.51] 3.05

$0.40[0.22-0.73]$

$0.70[0.55-0.88]$

B

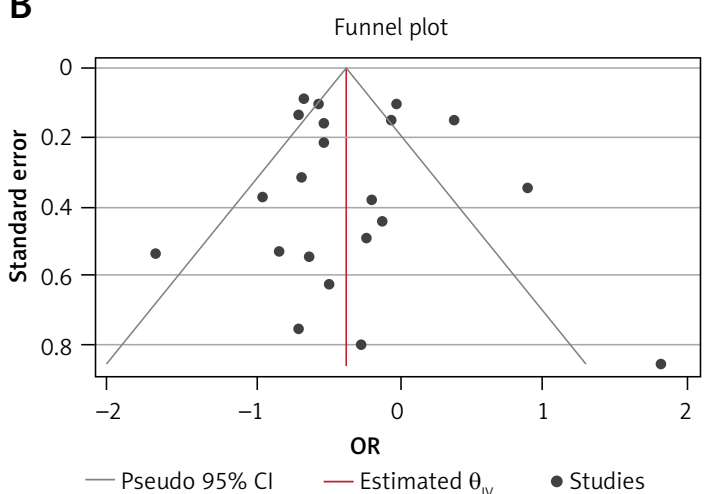

Figure 4. A - Forest plot showing the effect across the studies assessing pre-hospital $(n=18)$ and in-hospital $(n=3)$ use of statins for the death outcome (overall for each subgroup shown as red symbols). The overall effect is shown by the green symbol. B - Funnel plot showing the publication bias for all 21 studies which assessed the death outcome

severity could be due to the presence of diseases, such as hypertension, obesity, or lipid disorders, which are significant risk factors for worse

outcomes [10-12]. A study carried out in Denmark showed that recent statin use in COVID-19 patients was not associated with an effect on 
Amir Vahedian-Azimi, Seyede Momeneh Mohammadi, Farshad Heidari Beni, Maciej Banach, Paul C. Guest, Tannaz Jamialahmadi, Amirhossein Sahebkar

Leukocyte

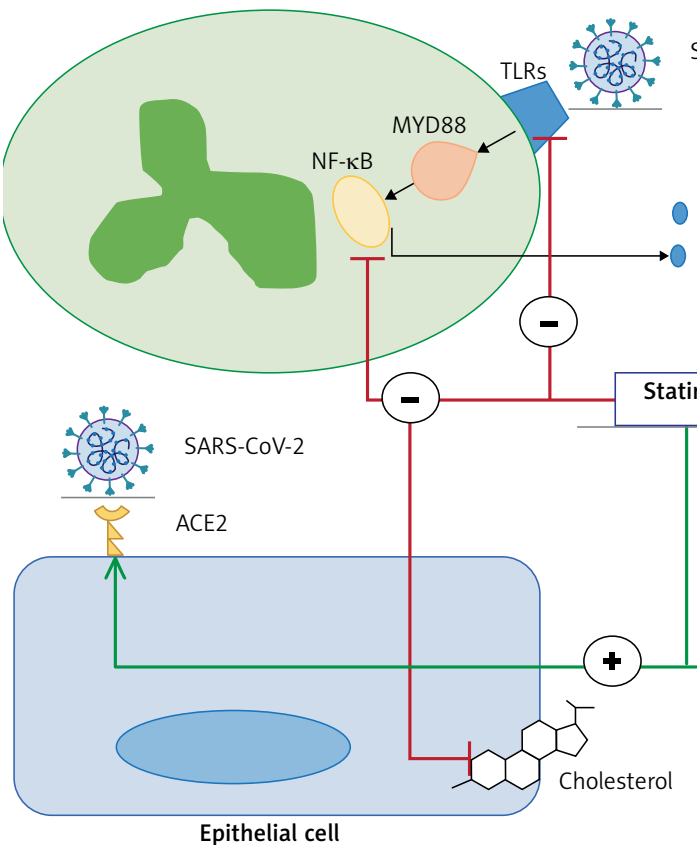

Plateled

SARS-CoV-2

Proinflammatory cytokines

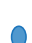

0 kines
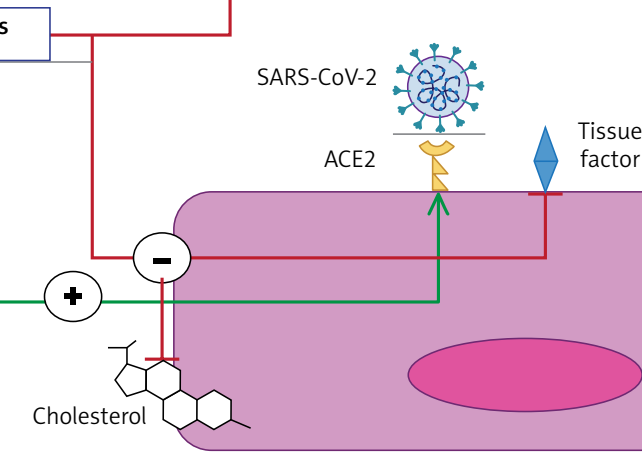

Endothelial cell

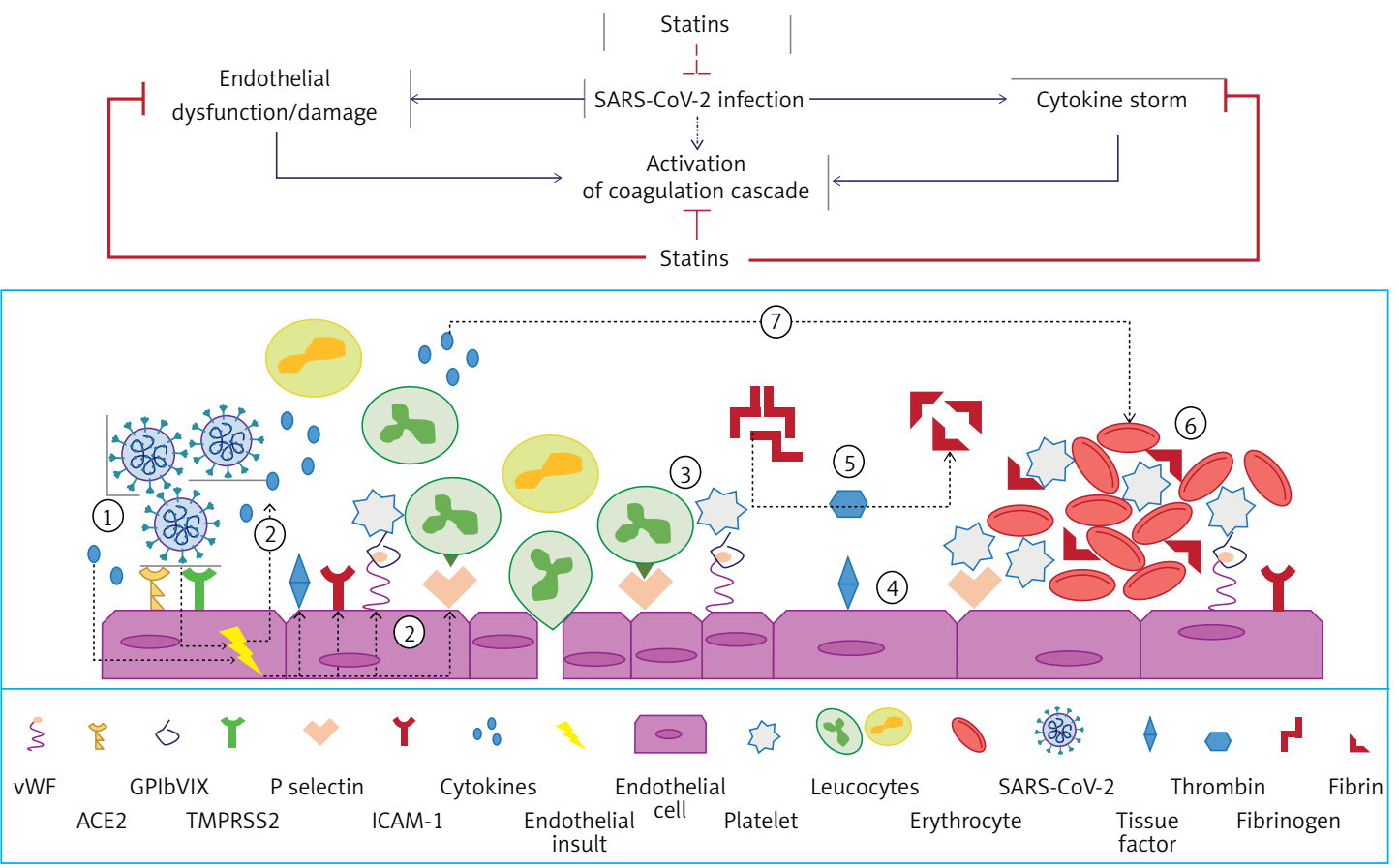

Figure 5. (Upper figure) Proposed mechanisms for the beneficial effects of statins in SARS-CoV-2 infection. Cellular pathways altered by statins during the course of infection in COVID-19 patients. Surface expression of ACE2 in epithelial and endothelial cells is enhanced by statins. Statins also mitigate the MYD88-NF-KB pathway and the ensuing proinflammatory cytokine response following interaction of SARS-CoV-2 with toll-like receptors (TLRs). The anti-thrombotic effects of statins are mediated by suppressive effects on tissue factor, and cytosolic phospholipase A2 (CPLA2)-induced thromboxane A2 (TXA2) synthesis and inactivated endothelial cells and platelets, respectively. Statins have also been suggested to decrease plasma membrane cholesterol content in the host cells, which can interfere with the replication of virus particles. (Lower figure) Proposed mechanisms for the anti-thrombotic effects of statins in SARS-CoV-2 infection SARS-CoV-2 induces activation of endothelial cells and the release of pro-inflammatory cytokines, which can lead to:1) over-expression of adhesion molecules (e.g. P-selectins, intercellular adhesion molecule 1 (ICAM-1), von Willebrand factor, $\alpha v \beta 3$ ) and further release of proinflammatory cytokines; 2) increased recruitment of leukocytes and platelets; 3 ) activated endothelial cells also express tissue factor, which activates factor VII, factor Xa, and thrombin formation; 4) fibrinogen is cleaved into fibrin by thrombin; 5) thrombin is essential for thrombus formation; 6)pro-inflammatory factors can also promote coagulation and accelerate platelet activation and thrombus formation; and 7)statins downregulate proinflammatory cytokine release from endothelial cells and mitigate coagulation cascade. Moreover, statins interfere with SARS-CoV-2 entry intoACE2expressing endothelial cells and ensuing endothelial activation (reproduced with permission from [18]) 
all-cause mortality or severe infection [51]. It could be that the potential benefit of statin-use was offset by the presence of co-morbidities. Although the researchers attempted to adjust for the potential effects of medication differences, they could not exclude a possible impact on the association between statin use and outcomes, or the presence of pre-existing co-morbidities. Here, we have attempted to address this in our subgroup analysis which compared the effects of pre- and in-hospital use of statins on COVID-19 outcomes. However, future studies should also include an assessment of whether or not pre-existing diseases are present.

The findings of this study should be interpreted by considering a number of limitations. First, the study did not allow any assessment of a causeand-effect relationship and, therefore, only associations are reported. Second, confounding factors cannot be excluded such as the pre-existing or post-diagnosis development of co-morbidities, including insulin resistance, ARDS, and coagulopathies, which may have affected some outcomes. Third, the findings have not been adjusted for concomitant medications. It should also to be emphasised, that based on the data from the included studies, we were not able to include the sub-analyses on the statin preparations and the investigated outcomes. Finally, our finding that in-hospital statin use was associated with decreased death compared to pre-hospital use did not reach significance. However, this is also likely to be due to the fact that there were only 3 studies [52-54] in the in-hospital use group and this was therefore likely to be statistically underpowered.

In conclusion, we showed that statin therapy might be useful in the context of a lower risk of the severe course of the COVID-19 with a reduction of ICU admission and mortality. Further studies are urgently needed to establish the role of statins in patients with COVID-19. Such studies should assess the importance of pre-existing medical conditions as well as medications on clinical outcomes. These findings also highlight the need for systematic clinical studies to assess both pre- and in-hospital use of statins as the best means of reducing COVID-19 disease severity and mortality. Successful randomised clinical studies proving that statins offer a viable therapeutic option for COVID-19 disease might pave the way for their rapid deployment in the field as needed, due to their low cost, high availability, and well-established safety and tolerability profiles. Furthermore, the knowledge that we have gained over the past several months regarding COVID-19 and repurposing existing therapeutics such as statins will provide important insights and strategic measures to successfully control future epidemics and pandemics.

\section{Conflict of interest}

Maciej Banach: speakers bureau: Amgen, DaichiiSankyo, Esperion, Herbapol, KRKA, MSD, Mylan, Novartis, Novo-Nordisk, Sanofi-Aventis, Servier; consultant to Abbott Vascular, Akcea, Amgen, Daichii Sankyo, Esperion, Lilly, MSD, Resverlogix, Sanofi-Aventis; Grants from Amgen, Mylan, Sanofi and Valeant. All other authors have no conflict of interest.

\section{References}

1. Worldometer: coronavirus cases. https://www.worldometers.info/coronavirus/\#countries (Accessed: 18.12.2020).

2. Johns Hopkins University and Medicine: Coronavirus Resource Center. https://coronavirus.jhu.edu/ (Accessed: 18.12.2020).

3. Worldometer: Current World Population. https://www.worldometers.info/world-population/ (Accessed: 18.12.2020).

4. Oliver SE, Gargano JW, Marin M, et al. The Advisory Committee on Immunization Practices' Interim Recommendation for Use of Pfizer-BioNTech COVID-19 Vaccine - United States, December 2020. MMWR Morb Mortal Wkly Rep 2020; 69: 1922-4.

5. Voysey M, Clemens SAC, Madhi SA, et al. Safety and efficacy of the ChAdOx1 nCoV-19 vaccine (AZD1222) against SARS-CoV-2: an interim analysis of four randomised controlled trials in Brazil, South Africa, and the UK. Lancet 2021; 397: 99-111.

6. BBC News: Covid: Moderna vaccine moves closer to US approval. https://www.bbc.co.uk/news/world-us-canada-55358334 (Accessed: 18.12.2020).

7. Zahedipour F, Hosseini SA, Sathyapalan T, et al. Potential effects of curcumin in the treatment of COVID-19 infection. Phytother Res 2020; 34: 2911-20.

8. Banach M, Penson PE, Fras Z, et al. Brief recommendations on the management of adult patients with familial hypercholesterolemia during the COVID-19 pandemic. Pharmacol Res 2020; 158: 104891.

9. Huang C, Wang Y, Li X, et al. Clinical features of patients infected with 2019 novel coronavirus in Wuhan, China. Lancet 2020; 395: 497-506.

10. Noor FM, Islam MM. Prevalence and associated risk factors of mortality among COVID-19 patients: a metaanalysis. J Commun Health 2020; 45: 1270-82.

11. Biswas M, Rahaman S, Biswas TK, Haque Z, Ibrahim B. Association of sex, age, and comorbidities with mortality in COVID-19 patients: a systematic review and meta-analysis. Intervirology 2020; doi: 10.1159/ 000512592

12. Moazzami B, Chaichian S, Kasaeian A, et al. Metabolic risk factors and risk of Covid-19: a systematic review and meta-analysis. PLoS One 2020; 15: e0243600.

13. Petrovic V, Radenkovic D, Radenkovic G, Djordjevic V, Banach M. Pathophysiology of cardiovascular complications in COVID-19. Front Physiol 2020; 11: 575600.

14. Rosenthal N, Cao Z, Gundrum J, Sianis J, Safo S. Risk factors associated with in-hospital mortality in a US national sample of patients with COVID-19. JAMA Network Open 2020; 3: e2029058-e.

15. Urbach D, Awiszus F, Leiß S, Venton T, De Specht AV, Apfelbacher C. Associations of medications with lower odds of typical COVID-19 symptoms: cross-sectional symptom surveillance study. JMIR Public Health Surveill 2020; 6: e22521. 
16. Ghati N, Roy A, Bhatnagar S, et al. Atorvastatin and aspirin as adjuvant therapy in patients with SARS-CoV-2 infection: a structured summary of a study protocol for a randomised controlled trial. Trials 2020; 21: 1-3.

17. Minz MM, Bansal M, Kasliwal RR. Statins and SARS-CoV-2 disease: current concepts and possible benefits. Diabetes Metab Syndr 2020; 14: 2063-7.

18. Ganjali S, Bianconi V, Penson PE, et al. Commentary: statins, COVID-19, and coronary artery disease: killing two birds with one stone. Metabolism 2020; 113: 154375

19. Sahebkar A, Serban C, Ursoniu S, et al. The impact of statin therapy on plasma levels of von Willebrand factor antigen: systematic review and meta-analysis of randomised placebo-controlled trials. Thromb Haemost 2016; 115: 520-32.

20. Katsiki N, Banach M, Mikhailidis DP. More good news on statins and COVID-19. Am J Cardiol 2020; 138: 127-8.

21. Radenkovic D, Chawla S, Pirro M, Sahebkar A, Banach M. Cholesterol in relation to COVID-19: should we care about it? J Clin Med 2020; 9: 1909.

22. Patrick L, Uzick M. Cardiovascular disease: C-reactive protein and the inflammatory disease paradigm: HMG-CoA reductase inhibitors, alpha-tocopherol, red yeast rice, and olive oil polyphenols. A review of the literature. $\mathrm{Al}$ tern Med Rev 2001; 6: 248-71.

23. Parizadeh SM, Azarpazhooh MR, Moohebati M, et al. Simvastatin therapy reduces prooxidant-antioxidant balance: results of a placebo-controlled cross-over trial. Lipids 2011; 46: 333-40.

24. Serban C, Sahebkar A, Ursoniu S, et al. A systematic review and meta-analysis of the effect of statins on plasma asymmetric dimethylarginine concentrations. Sci Rep 2015; 5: 9902.

25. Liberale L, Carbone F, Montecucco F, Sahebkar A. Statins reduce vascular inflammation in atherogenesis: a review of underlying molecular mechanisms. Int J Biochem Cell Biol 2020; 122: 105735.

26. Wang T, Wang F, Gou Z, et al. Using real-world data to evaluate the association of incretin-based therapies with risk of acute pancreatitis: a meta-analysis of 1324 515 patients from observational studies. Diabetes Obes Metab 2015; 17: 32-41.

27. Saragiotto BT, Yamato TP, Junior LCH, Rainbow MJ, Davis IS, Lopes AD. What are the main risk factors for running-related injuries? Sports Med 2014; 44: 1153-63.

28. Liberati A, Altman DG, Tetzlaff J, et al. The PRISMA statement for reporting systematic reviews and meta-analyses of studies that evaluate health care interventions: explanation and elaboration. J Clin Epidemiol 2009; 62: e1-34.

29. Hardy RJ, Thompson SG. A likelihood approach to metaanalysis with random effects. Stat Med 1996; 15: 619-29.

30. Higgins JP, Thompson SG. Quantifying heterogeneity in a meta-analysis. Stat Med 2002; 21: 1539-58.

31. Egger M, Smith GD, Schneider M, Minder C. Bias in meta-analysis detected by a simple, graphical test. BMJ 1997; 315: 629-34.

32. Begg CB, Mazumdar M. Operating characteristics of a rank correlation test for publication bias. Biometrics 1994; 50: 1088-101

33. Duval S, Tweedie R. Trim and fill: a simple funnel-plotbased method of testing and adjusting for publication bias in meta-analysis. Biometrics 2000; 56: 455-63.

34. Wang B, Van Oekelen O, Mouhieddine TH, et al. A tertiary center experience of multiple myeloma patients with COVID-19: lessons learned and the path forward. J Hematol Oncol 2020; 13: 94.
35. Krishnan S, Patel K, Desai R, et al. Clinical comorbidities, characteristics, and outcomes of mechanically ventilated patients in the State of Michigan with SARS-CoV-2 pneumonia. J Clin Anesth 2020; 67: 110005.

36. Subir R, Jagat JM, Kalyan KG. Pros and cons for use of statins in people with coronavirus disease-19 (COVID-19). Diabetes Metab Syndr 2020; 14: 1225-9.

37. Abdulrab S, Alkadasi B, Al-Maweri S, Halboub E, Alhadainy $\mathrm{H}$, Geerts $\mathrm{G}$. Statins-based prophylactic mouthwash and nasal spray may protect against coronavirus disease 2019. New Microbes New Infect 2020; 37 : 100751.

38. Wang Y, Yang W, Zhao X, Zhang R. Experimental study of the protective effect of simvastatin on lung injury in rats with sepsis. Inflammation 2018; 41: 104-13.

39. Nežić L, Škrbić R, Amidžić L, Gajanin R, Kuča K, Jaćević V. Simvastatin Protects cardiomyocytes against endotoxin-induced apoptosis and up-regulates survivin/NF-кB/ p65 expression. Sci Rep 2018; 8: 14652.

40. Teoh N, Farrell G. Statins as early therapy to mitigate COVID-19 (SARS-CoV-2)-associated ARDS and cytokine storm syndrome - time is of the essence. J Clin Trans Res 2020; 5: 227-9.

41. Bhaskar S, Sinha A, Banach M, et al. Cytokine storm in COVID-19 - immunopathological mechanisms, clinical considerations, and therapeutic approaches: the REPROGRAM consortium position paper. Front Immunol 2020; 11: 1648

42. Tsai PH, Lai WY, Lin YY, et al. Clinical manifestation and disease progression in COVID-19 infection. J Chin Med Assoc 2021; 84: 3-8.

43. Gorabi AM, Kiaie N, Bianconi V, et al. Antiviral effects of statins. Prog Lipid Res 2020; 79: 101054.

44. Reiner Ž, Hatamipour $M$, Banach $M$, et al. Statins and the COVID-19 main protease: in silico evidence on direct interaction. Arch Med Sci 2020; 16: 490-6.

45. Kow CS, Hasan SS. Meta-analysis of effect of statins in patients with COVID-19. Am J Cardiol 2020; 134: 153-5.

46. Grasselli G, Greco M, Zanella A, et al. Risk factors associated with mortality among patients with COVID-19 in intensive care units in Lombardy, Italy. JAMA Intern Med 2020; 180: 1345-55.

47. Yan $H$, Valdes AM, Vijay A, et al. Role of drugs used for chronic disease management on susceptibility and severity of COVID-19: a large case-control study. Clin Pharmacol Ther 2020; doi: 10.1002/cpt.2047.

48. Rossi R, Talarico M, Coppi F, Boriani G. Protective role of statins in COVID 19 patients: importance of pharmacokinetic characteristics rather than intensity of action. Intern Emerg Med 2020; 15: 1573-6.

49. Cariou B, Goronflot T, Rimbert A, et al. Routine use of statins and increased COVID-19 related mortality in inpatients with type 2 diabetes: results from the CORONADO study. Diabetes Metab 2021; 47: 101202.

50. Saeed O, Castagna F, Agalliu I, et al. Statin use and in-hospital mortality in diabetics with COVID-19. J Am Heart Assoc 2020; 9: e018475.

51. Butt JH, Gerds TA, Schou M, et al. Association between statin use and outcomes in patients with coronavirus disease 2019 (COVID-19): a nationwide cohort study. BMJ Open 2020; 10: e044421.

52. Zhang XJ, Qin JJ, Cheng X, et al. In-hospital use of statins is associated with a reduced risk of mortality among individuals with COVID-19. Cell Metab 2020; 32: 17687.e4.

53. Rodriguez-Nava G, Trelles-Garcia DP, Yanez-Bello MA, Chung CW, Trelles-Garcia VP, Friedman HJ. Atorvas 
tatin associated with decreased hazard for death in COVID-19 patients admitted to an ICU: a retrospective cohort study. Critical Care 2020; 24: 429.

54. Vahedian-Azimi A, Rahimibashar F, Najafi A, et al. Association of in-hospital use of statins, aspirin and renin-angiotensin-aldosterone inhibitors with mortality and ICU admission due to COVID-19. Adv Exp Med Biol 2021 (In Press).

55. Masana L, Correig E, Rodríguez-Borjabad C, et al. Effect of statin therapy on SARS-CoV-2 infection-related. Eur Heart J Cardiovasc Pharmacother 2020; doi: 10.1093/ ehjcvp/pvaa128.

56. Song SL, Hays SB, Panton CE, et al. Statin use is associated with decreased risk of invasive mechanical ventilation in COVID-19 patients: a preliminary study. Pathogens 2020; 9: 759.

57. Argenziano MG, Bruce SL, Slater CL, et al. Characterization and clinical course of 1000 patients with coronavirus disease 2019 in New York: retrospective case series. BMJ 2020; 369: m1996.

58. De Spiegeleer A, Bronselaer A, Teo JT, et al. The effects of ARBs, ACEls and statins on clinical outcomes of COVID-19 infection among nursing home residents. J Am Med Dir Assoc 2020; 21: 909-914.e2.

59. Dreher M, Kersten A, Bickenbach J, et al. The charac teristics of 50 hospitalized COVID-19 patients with and without ARDS. Deutsches Ärzteblatt International 2020; 117: 271.

60. Tan WY, Young BE, Lye DC, Chew DE, Dalan R. Statin use is associated with lower disease severity in COVID-19 infection. Sci Rep 2020; 10: 17458.

61. Daniels LB, Sitapati AM, Zhang J, et al. Relation of statin use prior to admission to severity and recovery among COVID-19 inpatients. Am J Cardiol 2020; 136: 149-55.

62. Gupta A, Madhavan MV, Poterucha TJ, et al. Association between antecedent statin use and decreased mortality in hospitalized patients with COVID-19. Res Sq 2020; rs.3.rs-56210 (Preprint); doi: 10.21203/rs.3.rs-56210/v1.

63. Cariou B, Goronflot T, Rimbert A, et al. Routine use of statins and increased mortality related to COVID-19 in inpatients with type 2 diabetes: results from the CORONADO study. Diabetes Metab 2020; doi: 10.1016/j.diabet.2020.10.001.

64. Peymani P, Dehesh T, Aligolighasemabadi F, et al. Statins in patients with COVID-19: a retrospective cohort study in Iranian COVID-19 patients. Res Sq 2020 (Preprint); doi: 10.21203/rs.3.rs-106030/v1

65. Rossi R, Talarico M, Coppi F, Boriani G. Protective role of statins in COVID 19 patients: importance of pharmacokinetic characteristics rather than intensity of action. Intern Emerg Med 2020; 15: 1573-76.

66. Zenga $\mathrm{H}$, Zhang $\mathrm{T}$, He $\mathrm{X}$, et al. Impact of hypertension on progression and prognosis in patients with COVID-19. medRxiv 2020.06.14.20125997.

67. Nguyen AB, Upadhyay GA, Chung B, et al. Outcomes and cardiovascular comorbidities in a predominantly African-American population with COVID-19. medRxiv 2020.06.28.20141929;

68. Ayed M, Borahmah AA, Yazdani A, Sultan A, Mossad A, Rawdhan $\mathrm{H}$. Assessment of clinical characteristics and mortality-associated factors in COVID-19 critical cases in Kuwait. Medical Principles and Practice 2021; 30: 185-92.

69. Nicholson CJ, Wooster L, Sigurslid HH, et al. Estimating risk of mechanical ventilation and mortality among adult COVID-19 patients admitted to Mass General Brigham: the VICE and DICE Scores. medRxiv [Preprint] 2020.09.14.20194670.
70. Lala A, Johnson KW, Januzzi JL, et al. Prevalence and impact of myocardial injury in patients hospitalized with COVID-19 infection. J Am Coll Cardiol 2020; 76: 533-46. 